\title{
Percepción de los estudiantes de medicina del ambiente educativo en una facultad con currículo tradicional (UCH-Chile) y otra con currículo basado en problemas (UNC-Argentina)
}

\author{
Gabriela Díaz-Véliz, Sergio Mora, Ricardo Bianchi, Pascual A. Gargiulo, Carolina Terán, Dorian Gorena, \\ José V. Lafuente-Sánchez, Jesús F. Escanero-Marcen
}

Objetivo. Evaluar y comparar la percepción que del ambiente educativo tienen los estudiantes de medicina de dos universidades iberoamericanas: Universidad de Chile (UCH) y Universidad Nacional de Cuyo (UNC), que desarrollan un currículo tradicional y un currículo basado en problemas, respectivamente.

Sujetos y métodos. Participaron 465 estudiantes: 232 de la UCH y 233 de la UNC. La distribución fue de 84 y 70 estudiantes para el primer curso, 77 y 97 para el tercero, y 71 y 66 para el quinto, respectivamente. Se aplicó el cuestionario DREEM, que consiste en 50 ítems, agrupados en cinco dimensiones: percepción de la enseñanza, percepción de los profesores, autopercepción académica, percepción de la atmósfera educativa y autopercepción social.

Resultados. Las puntuaciones totales fueron mayores en los tres cursos de la UNC. Resultaron similares en todos los cursos de ambas universidades, excepto en el quinto curso de la UCH. Respecto a la percepción acerca de los profesores, los estudiantes de quinto curso de la UCH mostraron las puntuaciones más bajas, mientras que los estudiantes del primer curso de la UNC tuvieron la mejor percepción. Resultados similares se obtuvieron en la autopercepción académica. La percepción del ambiente de aprendizaje fue mejor en la UNC y la autopercepción social tuvo puntuaciones similares en todos los cursos de ambas universidades.

Conclusiones. Las diferencias observadas entre ambas universidades podrían atribuirse a sus diferentes currículos. El currículo basado en problemas parece ser mejor valorado que el tradicional. Nuestro estudio corrobora la eficacia del cuestionario DREEM para identificar fortalezas y debilidades del currículo y para evaluar la calidad de la enseñanza en facultades de medicina.

Palabras clave. Ambiente educativo. Currículo. DREEM. Enseñanza. Estudiantes de medicina. Percepción de los estudiantes.

Medical students' perception of the educational environment in a faculty developing a traditional curriculum (UCH-Chile) and another with a problem based learning curriculum (UNC-Argentina)

Aim. To assess and compare the perception about the educational environment of medical students from two Latin-American universities, University of Chile (UCH) and National University of Cuyo (UNC), which develop a traditional curriculum and a problem based curriculum, respectively.

Subjects and methods. A transversal study was performed in 465 students: 232 from the UCH and 233 from the UNC. The distribution was $84 / 70$ for the first course, $77 / 97$ for the third one and $71 / 66$ for the fifth one, respectively. The DREEM questionnaire, which consists of 50 items, was applied. It covers 5 dimensions of the educational environment: perception about learning, perception about teachers, academic self-perception, perception about educational climate and social self-perception.

Results. Total DREEM scores were significantly higher in the UNC. Scores were similar in all courses from both universities, with the exception of fifth course UCH. Regarding their perception about teachers, students of the fifth course UCH showed the lowest score, whereas students of the first course UNC had the best perception. Similar results were obtained for the academic auto-perception; while the perception of the learning environment obtained higher scores in the three courses from the UNC. Social auto-perception was similar in all courses tested in this study.

Conclusions. Differences observed between both universities could be attributed to their different curricula. Problem based curriculum seems to be better appreciated than the traditional one. Our study corroborates the efficacy of the DREEM questionnaire to identify strengths and weaknesses of the curriculum and for the assessment of teaching quality in medical schools.

Key words. Curriculum. DREEM. Educational environment. Medical students. Student perceptions. Teaching.

Facultad de Medicina; Universidad de Chile; Santiago, Chile (G. DíazVéliz, S. Mora). Facultad de Ciencias Médicas; Universidad Nacional de Cuyo; Mendoza, Argentina (R. Bianchi, P.A. Gargiulo). Facultad de Medicina; Universidad de San Francisco Xavier; Sucre, Bolivia (C. Terán). Departamento de Salud Pública; Universidad Andina Simón Bolívar; Sucre, Bolivia (D. Gorena). Facultad de Medicina y Odontología; Universidad del País Vasco; Bilbao, España (J.V. LafuenteSánchez). Facultad de Medicina; Universidad de Zaragoza; Zaragoza, España (J.F. Escanero-Marcen).

Correspondencia:

Profa. Gabriela Díaz Véliz. Programa de Farmacología Molecular y Clínica. Instituto de Ciencias Biomédicas. Facultad de Medicina. Universidad de Chile. P.0. Box 16038. Santiago-9, Chile.

E-mail:

gdiaz@med.uchile.cl

Trabajo realizado bajo el auspicio de la Agencia Española de Cooperación Internacional y Desarrollo (AECID), proyecto B012377/07.

(c) 2011 Educación Médica 


\section{Introducción}

En las teorías de aprendizaje, el ambiente educativo es un concepto que adquiere cada vez mayor relevancia por su impacto en el proceso de enseñanzaaprendizaje. Genn y Harden [1] utilizan el término climate para referirse a la atmósfera global y característica de la clase y de la facultad. Estos autores atribuyen a Murray (1938) y a Pace y Stern (1958) la utilización del término 'presión ambiental' para referirse a las características presiones, situaciones de estrés, recompensas e influencias que caracterizan a un ambiente educativo.

Desde los años sesenta se han utilizado diversos instrumentos para evaluar el ambiente educativo. En 1961, Hutchins [2] creó el Medical School Environment Index (MSEI), 'uno de los primeros instrumentos específicamente desarrollados para la medición del ambiente en educación médica' [3]. El mencionado instrumento consta de 180 ítems, agrupados en 18 subescalas o dimensiones. Posteriormente se han desarrollado algunos otros [3-6] que, al no tener en cuenta las innovaciones curriculares y el cambio del proceso de enseñanza-aprendizaje (centrado ahora en el estudiante), han quedado pronto obsoletos. Estimulados por las sugerencias de Genn y Harden [1], Roff et al [7] desarrollaron y validaron el cuestionario DREEM (Dundee Ready Educational Environment Measure), aplicado en este estudio. Para su desarrollo se utilizó una metodología estándar y un panel Delphi de 100 educadores y 1.000 estudiantes de profesiones de la salud de todo el mundo, ya que se diseñó como un inventario de diagnóstico universal [8].

La aplicación del cuestionario DREEM permite generar el perfil de una institución evaluando sus fortalezas y debilidades tal y como son percibidas por los estudiantes de una determinada cohorte. También permite tener un diagnóstico para actuar sobre las deficiencias detectadas a fin de incrementar la calidad curricular, y realizar análisis comparativos acerca del medio ambiente educativo entre dos o más universidades o entre distintas cohortes de una misma institución, y correlacionar los resultados obtenidos con el desempeño académico. En general, la percepción que los estudiantes tienen del ambiente donde estudian puede ejercer un impacto significativo sobre la efectividad del aprendizaje, el progreso académico y la sensación de bienestar.

El objetivo del presente trabajo consiste en evaluar y comparar la percepción que tienen los estudiantes del ambiente educativo, utilizando el cuestionario DREEM [7], de dos facultades de medicina en dos universidades iberoamericanas con distinto currículo: la Universidad de Chile (UCH), con un currículo tradicional, y la Universidad Nacional de Cuyo (Mendoza, Argentina) (UNC), con un currículo que ha introducido como seña de identidad el aprendizaje basado en problemas.

\section{Sujetos y métodos}

\section{Muestra de estudio}

Este trabajo corresponde a un estudio de corte transversal, realizado en el año 2008. El cuestionario DREEM se aplicó a 465 estudiantes: 232 de la UCH y 233 de la UNC. La distribución de estudiantes fue de 84 y 70 para el primer curso, 77 y 97 para el tercero y 71 y 66 para el quinto, respectivamente. Se realizó una medición única en el aula de clase a todos los alumnos presentes que aceptaron participar tras la explicación del cuestionario, de los motivos y de los objetivos del trabajo planteado. Los alumnos que lo desearon pudieron abandonar la realización del test en cualquier momento sin explicación alguna. En ambos casos se trata de estudios oficiales en universidades públicas. En ninguno de los dos casos hay otras ofertas en igualdad de condiciones que hiciera necesario determinar las peculiaridades por las cuales los alumnos optaron por una u otra en función de la metodología.

\section{Herramienta utilizada}

El cuestionario DREEM consta de 50 ítems valorados mediante una escala tipo Likert de 5 puntos (0: muy en desacuerdo; 1 : parcialmente en desacuerdo; 2: inseguro/dudoso; 3: parcialmente de acuerdo; 4: totalmente de acuerdo). Así, altas puntuaciones totales indican una evaluación más positiva en cada aspecto del ambiente educativo. Se debe hacer notar que nueve ítems del cuestionario requieren una codificación inversa.

Los 50 ítems cubren conceptos relevantes y se agrupan en cinco dimensiones:

1. Percepción de la enseñanza (12 ítems).

2. Percepción acerca de los profesores (11 ítems).

3. Autopercepción académica (8 ítems).

4. Percepción de la atmósfera educativa (12 ítems). 5. Autopercepción social (7 ítems).

Al sumar las puntuaciones de los 50 ítems se obtiene un máximo total de 200. De acuerdo con los resultados obtenidos al sumar las puntuaciones alcanzadas por los individuos que respondieron al cuestionario, se puede determinar que puntuaciones totales entre 0 y 50 significan que el ambiente 
debe considerarse como muy pobre; entre 51 y 100 se considera que el ambiente tiene bastantes problemas; entre 101 y 149 se aprecia como adecuado, ya que presenta más aspectos positivos que negativos, y cuando supera los 150 puntos se estima que el clima educativo es excelente.

El DREEM fue traducido por uno de los autores y, posteriormente, se sometió a un análisis de comprensión por parte del grupo participante, al objeto de utilizar la misma herramienta en los diferentes países participantes. Después se comparó con la versión española del DREEM realizada por Deza [7] y no se encontraron variaciones que pudieran inducir a interpretaciones diferentes.

\section{Tratamiento estadístico}

Para procesar la información los datos se introdujeron en una hoja Excel y se analizaron con el programa GraphPad Prism v. 5, utilizando la prueba no paramétrica de Kruskall-Wallis para comparar las puntuaciones de los diferentes cursos de las dos universidades involucradas en este estudio, y la prueba no paramétrica $U$ de Mann-Whitney para comparar pares de grupos. Se trabajó con un nivel de significación $\alpha=0,05$.

\section{Resultados}

\section{Puntuaciones totales}

Según se aprecia en la figura 1, las puntuaciones totales del cuestionario DREEM fueron significativamente mayores en los tres cursos de la UNC comparados con la UCH. En esta última se observa una disminución significativa en la puntuación del 'ambiente educativo’ en los estudiantes de quinto curso respecto de los cursos inferiores de la misma universidad. En la tabla se aprecia que el número de individuos que perciben que el ambiente está lleno de problemas en la UCH es mayor que en la UNC (medias de 10-9-34\% y 0-2-8\%, respectivamente, para los tres cursos estudiados). En cambio, para la percepción de excelencia del ambiente educativo se observa que ésta es mayor en la UNC en comparación con la UCH, en los tres cursos (medias de 5336-27\% y 19-27-4\%, respectivamente).

La puntuación total se ha obtenido sumando las puntuaciones parciales para cada una de las cinco dimensiones, por lo que se ha analizado cada una de ellas con el fin de determinar cuál es más relevante en marcar las diferencias totales observadas entre ambas universidades.
Figura 1. Puntuaciones totales del cuestionario DREEM, expresadas como promedio \pm error estándar, según curso y universidad estudiada. a $p<0,05$ comparando el mismo curso en ambas universidades; ${ }^{\mathrm{b}} p<0,05$ al comparar cursos de una misma universidad.

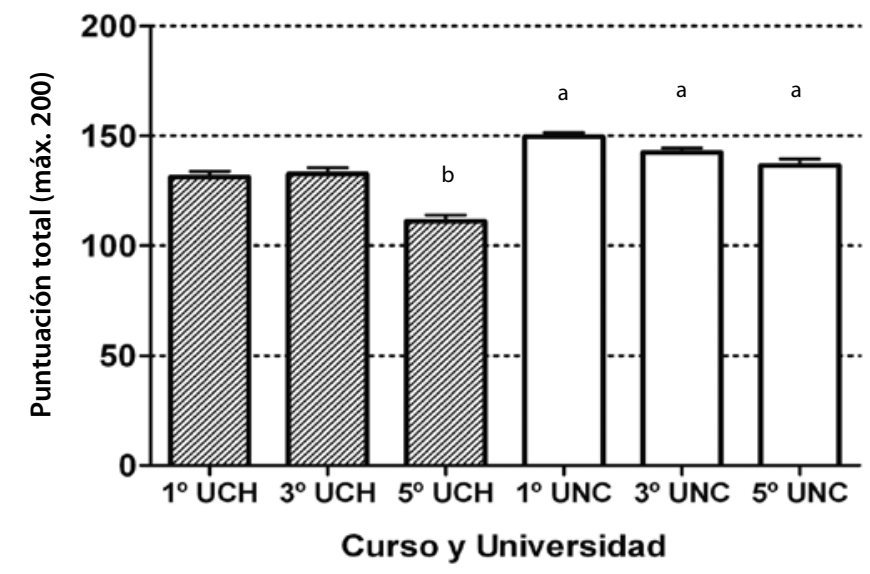

\begin{tabular}{|l|c|c|c|c|c|c|c|}
\hline & Maximo & \multicolumn{4}{|c|}{ Universidad de Chile } & \multicolumn{3}{c|}{ Universidad Nacional de Cuyo } \\
& posible & $1^{\circ}$ & $3^{\circ}$ & $5^{\circ}$ & $1^{\circ}$ & $3^{\circ}$ & $5^{\circ}$ \\
\hline PUNTUACION & 200 & 131,2 & 132,6 & 111,3 & 149,6 & 142,4 & 136,6 \\
\hline TOTAL DREEM & $100 \%$ & $65,60 \%$ & $66,30 \%$ & $55,60 \%$ & $74,8 \%$ & $71,2 \%$ & $68,2 \%$ \\
\hline
\end{tabular}

Figura 2. Percepción del aprendizaje. Puntuaciones (mediana y rango) para cada uno de los cursos analizados en la UCH y la UNC. a $p<0,05$ comparando el mismo curso en ambas universidades; ${ }^{b} p<0,05$ al comparar cursos de una misma universidad.

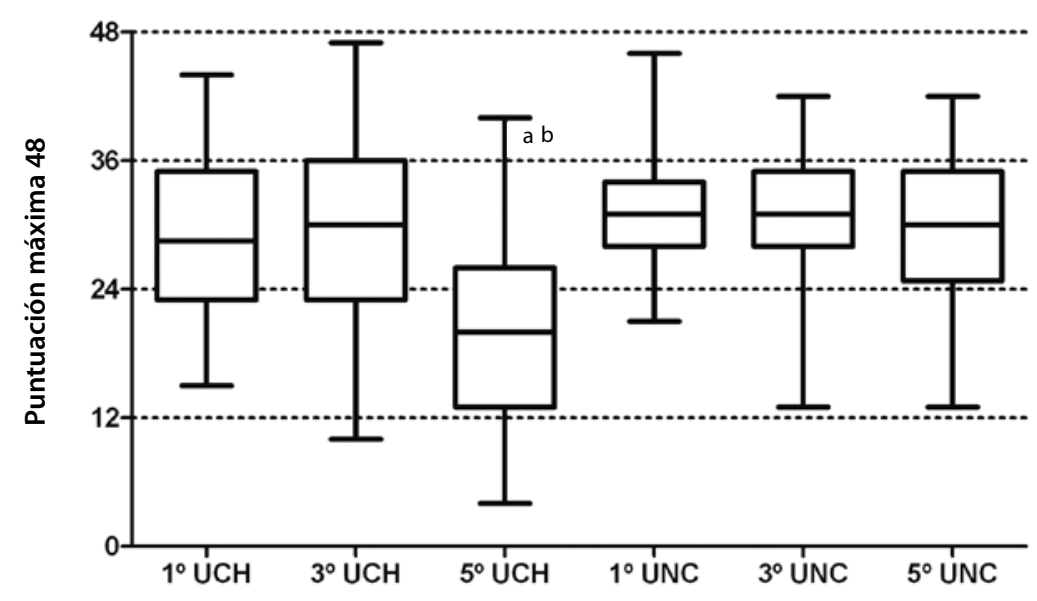

\section{Percepción de la enseñanza}

La figura 2 presenta las puntuaciones obtenidas en la dimensión 'percepción de la enseñanza', que resultan 
Tabla. Puntuaciones totales del cuestionario DREEM.

\begin{tabular}{|c|c|c|c|c|c|c|}
\hline & \multicolumn{3}{|c|}{ Universidad de Chile } & \multicolumn{3}{|c|}{ Universidad Nacional de Cuyo } \\
\hline & $\begin{array}{l}\text { Curso 1.ㅇ } \\
(n=84)\end{array}$ & 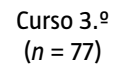 & $\begin{array}{l}\text { Curso 5.o } \\
(n=71)\end{array}$ & $\begin{array}{c}\text { Curso 1.ㅇ } \\
(n=70)\end{array}$ & $\begin{array}{l}\text { Curso } 3.9 \\
(n=97)\end{array}$ & $\begin{array}{l}\text { Curso 5.ㅇ } \\
(n=66)\end{array}$ \\
\hline Muy pobre $(<50)$ & $0 \%$ & $0 \%$ & $0 \%$ & $0 \%$ & $0 \%$ & $0 \%$ \\
\hline Lleno de problemas (51-100) & $10 \%$ & $9 \%$ & $34 \%$ & $0 \%$ & $2 \%$ & $8 \%$ \\
\hline Más positivo que negativo (101-149) & $71 \%$ & $64 \%$ & $62 \%$ & $47 \%$ & $62 \%$ & $65 \%$ \\
\hline Excelente (150-200) & $19 \%$ & $27 \%$ & $4 \%$ & $53 \%$ & $36 \%$ & $27 \%$ \\
\hline
\end{tabular}

ser muy similares en todos los cursos de ambas universidades, con excepción del quinto de la $\mathrm{UCH}$, en el cual la percepción es significativamente menor que en el resto. La mediana de la puntuación de los estudiantes del quinto curso de la UCH (intervalo: 12-24) se encuentra en el rango correspondiente a 'el aprendizaje es considerado negativamente', según McAleer y Roff [9]. Con el objetivo de identificar cuáles son los aspectos que constituyen debilidades del ambiente educativo y que requieren corrección, se ha calculado el promedio de respuestas en cada ítem. Aquellos ítems con un promedio inferior a 2,0 se consideran áreas muy problemáticas, mientras que un valor superior o igual a 3,5 señala aspectos realmente positivos [9]. Según la percepción de los estudiantes de quinto curso de la $\mathrm{UCH}$, casi todos los ítems correspondientes a este dominio proporcionan valores preocupantes. Por otra parte, 'la programación horaria está bien organizada' y 'la enseñanza sobreenfatiza el aprendizaje de hechos concretos' (éste es uno de los nueve ítems del cuestionario que requieren una codificación inversa) han sido los dos ítems con una baja puntuación en todos los cursos de ambas universidades. En este dominio no se observan ítems con valores mayores a 3,5 (excelentes) en ningún curso de ambas universidades.

\section{Percepción de los profesores}

En esta subescala, los estudiantes de quinto curso de la UCH conceden las puntuaciones mas bajas (Fig. 3). Por otra parte, los estudiantes del primer curso de la UNC presentan una muy buena puntuación media, mayor a la de los estudiantes del mismo curso de la $\mathrm{UCH}$, e incluso mejor que en los otros cursos de la UNC, encontrándose en el rango superior correspondiente a 'profesores modelo', según McAleer y Roff [9]. En el análisis de los ítems sólo se aprecia un ítem negativo (inferior a 2), 'los profesores son autoritarios' (requiere codificación inversa), que corresponde al quinto curso de la UCH. Los estudiantes del primer curso de la UNC conceden puntuaciones sobre 3,0 en casi todos los ítems; incluso el ítem 'los profesores están bien preparados' obtuvo una puntuación excelente.

\section{Autopercepción académica}

Una vez más, los estudiantes de quinto curso de la $\mathrm{UCH}$ han sido los que han otorgado la puntuación más baja dentro de su universidad y respecto al mismo curso de la UNC (Fig. 4). También en el primer curso, la autopercepción es significativamente mayor en los estudiantes de la UNC que en los de la UCH. En el análisis de los ítems se observa que los estudiantes de quinto curso de la UCH muestran tener problemas con el ítem 'soy capaz de memorizar todo lo que necesito' (media: 1,6), en tanto que los estudiantes de primer curso lo presentan con el ítem 'las estrategias de aprendizaje que antes me funcionaron, continúan funcionando' (media: 1,8). Los ítems a los que los estudiantes de ambas universidades conceden la mejor percepción son 'considero que me están formando bien para mi profesión' (media: 3,6), 'confío en aprobar este curso' y 'he aprendido mucho acerca de empatía en mi profesión' (medias por encima de 3 en todos los casos).

\section{Percepción del ambiente}

Los estudiantes de la UNC, en los tres cursos, se muestran significativamente más satisfechos que los estudiantes de la $\mathrm{UCH}$ respecto al ambiente de aprendizaje (Fig. 5). Además, los estudiantes de quinto 
curso de la UCH señalan una peor percepción de la atmósfera de estudio que los estudiantes de cursos inferiores de la misma universidad. En general, los estudiantes de primer curso de la UNC tienen una percepción muy positiva en casi todos los ítems de este dominio (medias por encima de 3 ), excepto en el ítem 'la escuela tiene los tiempos adecuadamente programados', el cual es evaluado negativamente en todos los cursos de ambas universidades (entre 1,3 y 1,7). Los ítems 'el placer de estudiar medicina es mayor que el estrés que esto produce' y 'el ambiente motiva mi aprendizaje' son percibidos de forma negativa por los estudiantes de quinto curso de la $\mathrm{UCH}$ (medias de 1,9).

\section{Autopercepción social}

Respecto a la autopercepción social (Fig. 6), pueden observarse puntuaciones medias muy similares en todos los cursos de ambas universidades, apreciándose la única diferencia significativa en el tercer curso, en el que la percepción en la UNC fue significativamente mayor que en los estudiantes de la UCH. Los estudiantes de ambas universidades tienen una excelente percepción en varios de los ítems de este dominio, a excepción del que señala 'existe un buen sistema de apoyo para los estudiantes que se estresan', el cual tiene una puntuación media muy baja por parte de los estudiantes de ambas universidades (en torno a 1,5), lo mismo que el ítem 'estoy muy cansado para disfrutar del curso' (en torno a 1,3), que requiere codificación inversa, mostrando una percepción negativa por parte de los estudiantes de ambas universidades.

\section{Discusión}

Los resultados totales obtenidos en este estudio reflejan que los estudiantes de la UNC tienen una mejor percepción del ambiente educativo que los estudiantes de la UCH. La puntuación total se mantuvo o disminuyó levemente en el tercer curso (UCH: 65,6 a 66,3\%; UNC: 74,8 a 71,2\%), pero empeoró significativamente en los estudiantes de quinto curso (UCH: $55,6 \%$; UNC: $68,2 \%$ ), lo cual constituye una señal de necesidad de mejora.

En términos generales, los resultados totales obtenidos en la UCH (promedio total: 55,6-66,3\%) se encuentran dentro del rango referido en investigaciones similares: campus de Huesca de la Universidad de Zaragoza, 61,2\% [10]; Tailandia, 68,7\% [11]; Reino Unido, 66,2\% [7]; Nepal, 65,0\% [12]; Nigeria,
Figura 3. Percepción acerca de los profesores. Puntuaciones (mediana y rango) para cada uno de los cursos analizados en la UCH y la UNC. a $p<0,05$ comparando el mismo curso en ambas universidades; ${ }^{\mathrm{b}} p<0,05$ al comparar cursos de una misma universidad.

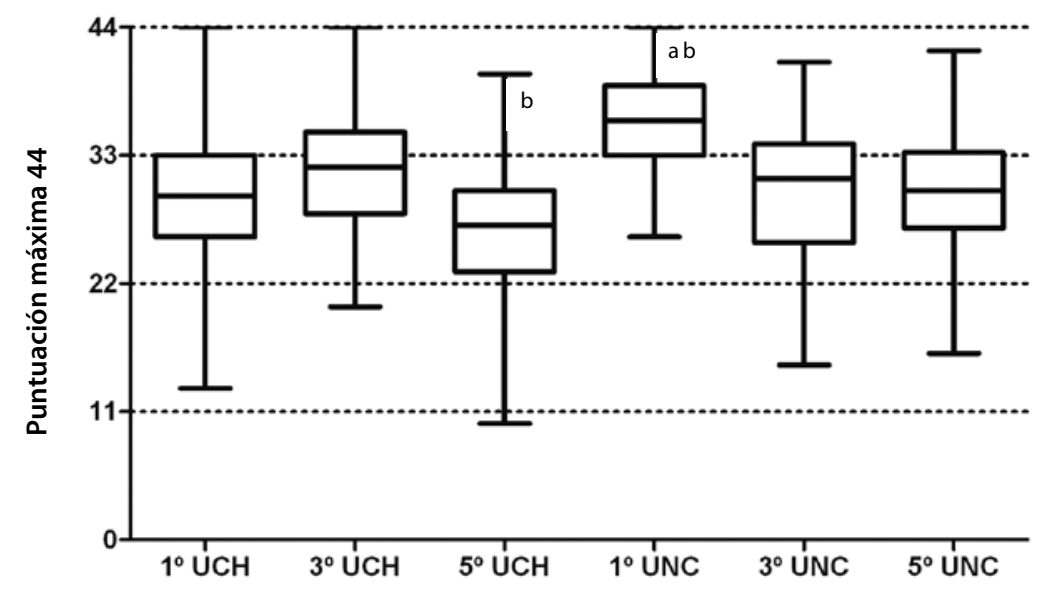

Figura 4. Autopercepción académica. Puntuaciones (mediana y rango) para cada uno de los cursos analizados en la UCH y la UNC. a $p<0,05$ comparando el mismo curso en ambas universidades; ${ }^{b} p<0,05$ al comparar cursos de una misma universidad.

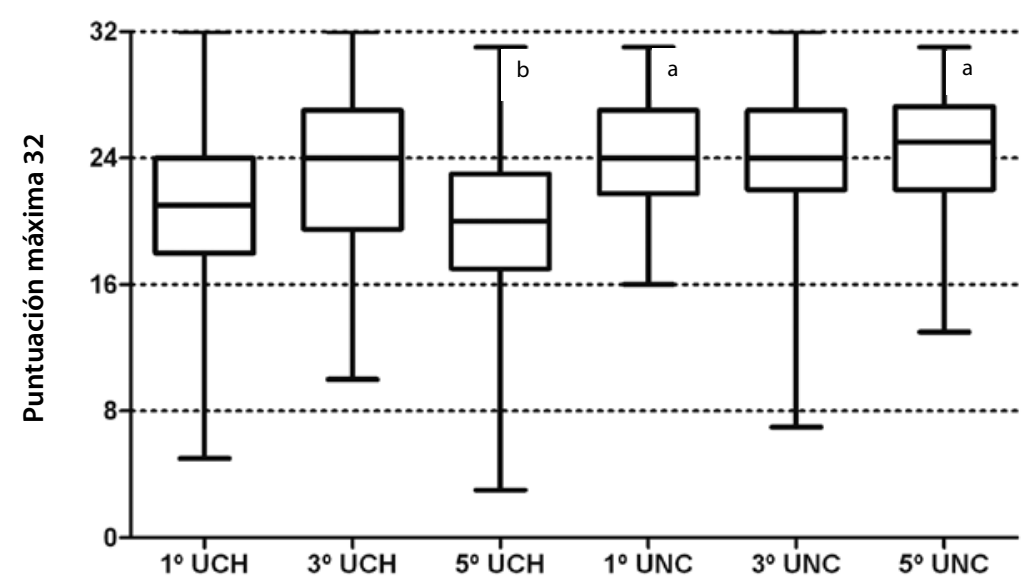

59,0\% [12]; Trinidad, 55,0\% [13] (promedios totales). En cambio, los datos de la UNC, en general, se encuentran sobre el nivel de la mayoría de las referencias indicadas (promedio total: $68,2-74,8 \%$ ). Algunas de las diferencias observadas entre ambas universidades podrían estar determinadas por el currículo, lo que sugiere que el tipo de formación que reciben los estudiantes de la UNC (aprendizaje basado en problemas) resultaría mejor valorado. 
Figura 5. Percepción de la atmósfera. Puntuaciones (mediana y rango) para cada uno de los cursos analizados en la UCH y la UNC. a $p<0,05$ comparando el mismo curso en ambas universidades; ${ }^{b} p<0,05$ al comparar cursos de una misma universidad.

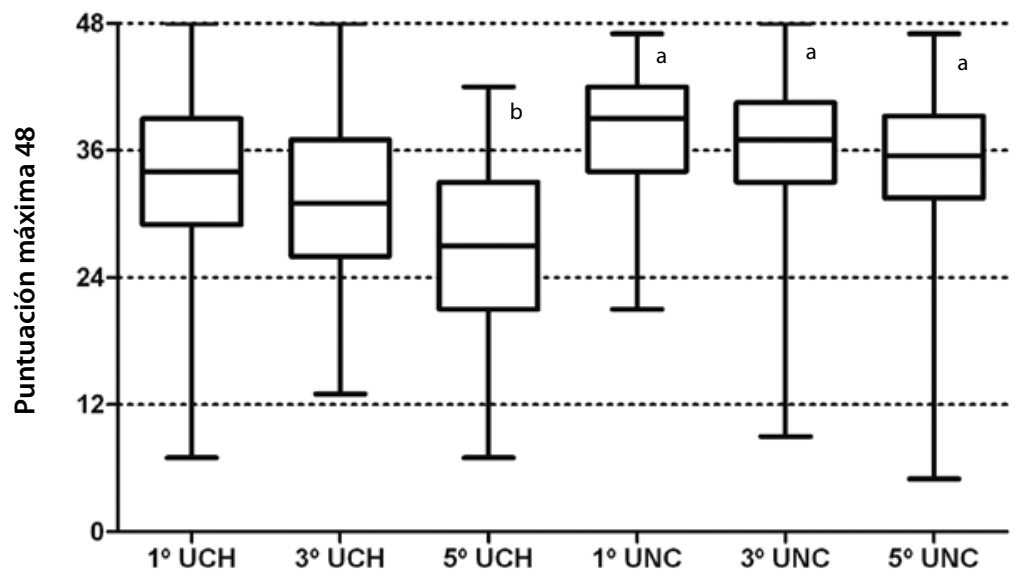

Figura 6. Autopercepción social. Puntuaciones (mediana y rango) para cada uno de los cursos analizados en la UCH y la UNC. a $p<0,05$ comparando el mismo curso en ambas universidades.

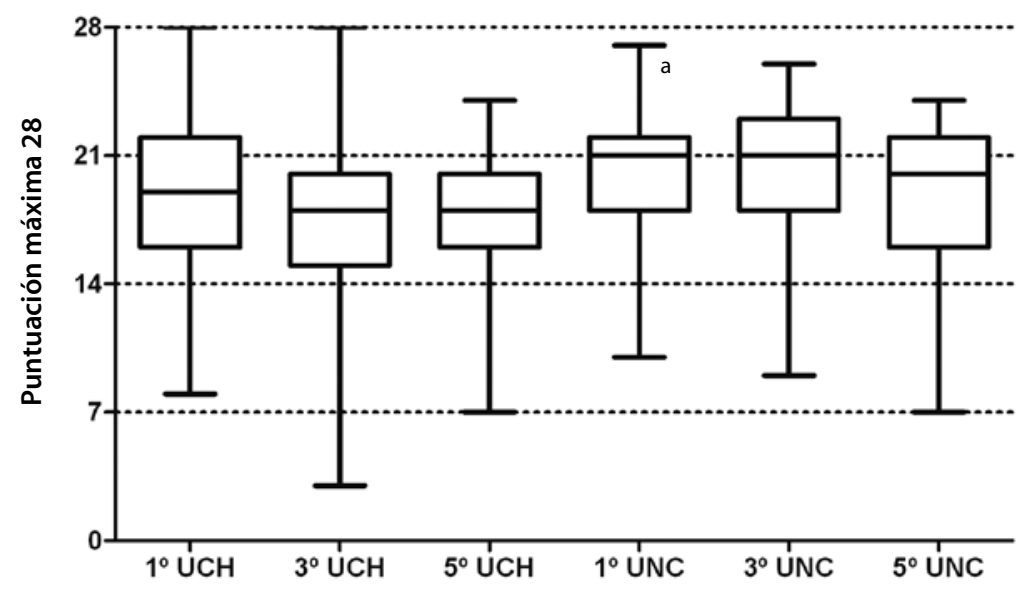

$\mathrm{Al}$ analizar las puntuaciones obtenidas en cada dominio, se observa que la satisfacción general en cuatro de los cinco dominios disminuyó significativamente en los estudiantes de quinto curso de la $\mathrm{UCH}$, situación que ya se ha descrito en investigaciones realizadas en otros contextos [7,10,14]. Este hecho se justifica considerando que los estudiantes pierden algo de la neutralidad que tienen al ingreso en la universidad, mostrándose más críticos con el ambiente académico a medida que avanzan en sus estudios [14]. Estos datos están respaldados por Till [15], quien utilizando el DREEM, explora el ambiente 'actual' y, a continuación, el que considera 'ideal', comunicando que las mayores discordancias en las puntuaciones entre lo considerado 'ideal' y las percepciones reales exploradas ('actual') tienen lugar en los alumnos de tercero frente a los de primero y segundo en su centro (Canadian Memorial Chiropractic College), con cuatro cursos en el currículo. Otra explicación posible es que el ambiente de la enseñanza clínica que incide plenamente en los alumnos de quinto curso resulta menos propicio. A este respecto, en un estudio sobre el currículo oculto, Lempp y Seale [16] indicaron que una de las principales vías por la cuales los estudiantes aprenden la importancia de la jerarquía en medicina es a través de un tipo de enseñanza que implica humillación y que tiene lugar principalmente durante los años clínicos, donde la planificación deja mucho que desear y el azar gobierna la enseñanza. En esta línea, los ítems 'el placer de estudiar medicina es mayor que el estrés que esto produce' y 'el ambiente motiva mi aprendizaje', percibidos de forma negativa por los estudiantes de quinto de la $\mathrm{UCH}$, corroboran dicha afirmación. Sin embargo, dado que el DREEM explora la percepción de los estudiantes, este análisis debe mencionar algunas de las deficiencias que han puesto de manifiesto diferentes autores [17-19] en la enseñanza clínica. Así, Irby [17] indica que los estudiantes de clínicas adolecen de falta de tiempo para la reflexión, para establecer conexiones con la información proporcionada en los cursos básicos, para reestructurar el conocimiento que ya poseen y para comprometerse en la resolución de los problemas de los pacientes bajo su cuidado. Por otra parte, Irby señala, además, que las expectativas de los estudiantes de clínicas no son homogéneas y se quejan de la falta de retroalimentación sobre su aprendizaje y de los modelos y marcos a los que se enfrentan, que no siempre son los más adecuados. El panorama puede ser bastante preocupante porque muchos de los profesionales ocupados en la enseñanza no son conscientes de su papel como modelos en lo que al cuidado de los pacientes se refiere. Además, para la enseñanza clínica pueden contratarse profesionales con muy diferente nivel o bagaje preparatorio para la enseñanza. Estas apreciaciones también han sido realizadas por otros autores [18,19]. Los estudiantes de la UNC muestran puntuaciones medias que se mantienen constantes a lo largo de los tres cursos estudiados. Únicamente en la percepción acer- 
ca de los profesores se observó un aumento significativo en los alumnos de primer curso.

Los estudiantes de quinto curso de la $\mathrm{UCH}$, comparados con los del mismo curso de la UNC, mostraron tener una peor percepción en nueve de los 12 ítems referidos a la enseñanza, alcanzando cifras muy preocupantes que requieren un análisis profundo por parte de la institución. Esta subescala, la de percepción académica, junto con la de la atmósfera, son las determinantes de las mayores diferencias en los alumnos de quinto curso de ambas universidades. En cambio, no existen diferencias en la subescala de la autopercepción social. Se ha sugerido [20] que podrían existir algunas deficiencias para la socialización en los alumnos con un sistema basado en la solución de problemas debido a que, desde el principio, son repartidos en pequeños grupos frente a las actividades de curso entero en los currículos tradicionales. En ambas universidades, y en todos los cursos, los estudiantes manifestaron tener problemas con la programación horaria y con el mayor énfasis puesto en los hechos concretos, ítems que se quedaron con una puntuación inferior a 2,0.

En la valoración del profesorado, y de acuerdo con la opinión de los estudiantes de quinto curso, en la UCH los profesores no realizan retroalimentación, ni hacen una crítica constructiva, ni dan ejemplos claros, mientras que en la UNC, en los cursos más avanzados, los profesores se molestan más en las clases, se irritan con los estudiantes y pierden la paciencia frente a los enfermos. En ambas universidades, los profesores se vuelven autoritarios.

Respecto a las autopercepciones académicas, los estudiantes de quinto curso de ambas universidades bajan sus puntuaciones en los ítems correspondientes a la percepción sobre si los están formando bien, notablemente más en la UCH. Los estudiantes de quinto curso en esta facultad se sienten incapaces de memorizar todo lo que necesitan, lo cual puede indicar que la metodología empleada sigue siendo enciclopedista y centrada en el docente; incluso deja de parecerles relevante lo que aprenden y dudan de que los estén formando bien para su profesión. Es importante llamar la atención sobre la duda que los estudiantes de primer curso de la UCH tienen acerca de la utilidad del aprendizaje del curso anterior. Si se tiene en cuenta que en el curso anterior se encontraban cursando la enseñanza secundaria, este resultado es un indicador de la posible escasa adecuación entre la enseñanza secundaria y la universitaria. Este hecho no se observa en la UNC.

En relación con la atmósfera de aprendizaje, las puntuaciones señalan que todos los estudiantes perciben que la facultad no tiene los tiempos ade- cuadamente programados, lo cual concuerda con la percepción negativa acerca de que la programación horaria esté bien organizada. Resulta preocupante comprobar que los estudiantes de quinto curso de la $\mathrm{UCH}$ encuentran decepcionante la experiencia de aprendizaje y que disminuye el placer de estudiar medicina en relación al estrés que ello significa, a la vez que dudan de que el ambiente estimule su aprendizaje. Algo similar se ha observado recientemente en un estudio realizado en las universidades españolas de Zaragoza, Bilbao y Huesca [10].

Finalmente, con respecto a la autopercepción social, los estudiantes consideran que no hay un buen sistema de apoyo cuando se estresan, reconocen estar muy cansados como para disfrutar de su curso, y los de quinto de la $\mathrm{UCH}$ reconocen que a veces se aburren.

En general, como ya han indicado algunos autores [21-23], el cuestionario DREEM es una herramienta eficaz para diagnosticar áreas débiles en el currículo. Constituye una pieza importante para el seguimiento de la calidad de la enseñanza en las facultades de medicina y, como en este caso para la $\mathrm{UCH}$, para el establecimiento de una línea base de implementación del nuevo currículo, proceso en el cual se encuentran inmersos. Respecto a los resultados obtenidos en ambas facultades, especialmente en la UCH, llama la atención que los estudiantes que han permanecido más tiempo en la facultad se muestran menos satisfechos con el ambiente educativo $[12,24]$. Esto podría justificarse por dos hechos: uno, que los estudiantes se hacen más críticos a medida que avanzan en sus estudios, y otro, que el ambiente empeora durante la enseñanza clínica. Por otra parte, los resultados obtenidos han sido más elevados en la UNC que en la UCH, y siendo la enseñanza basada en problemas el aspecto más diferenciador, deben atribuirse a ésta los mencionados resultados. De todas maneras, las bajas puntaciones obtenidas en algunos ítems de las diferentes subescalas del DREEM requieren acciones correctoras en ambas facultades. Comparativamente, de manera global, las puntuaciones fueron más elevadas en la UNC en todos los cursos, siendo especialmente relevantes en quinto curso debido a las mayores puntuaciones otorgadas a las subescalas concernientes a enseñanza académica y atmósfera de aprendizaje.

\section{Bibliografía}

1. Genn JM, Harden RM. What is medical education here really like? Suggestions for action research studies of climates of medical education environments. Med Teach 1986; 8: 111-24.

2. Hutchins EB. The 1960 s medical school graduate: his perceptions of his faculty, peers, and environment. J Med Educ 1961; 36: 322-9. 
3. Riquelme A, Fuentes G, Jeria A, Méndez I, Aranís C, Larios $\mathrm{G}$, et al. Ambiente educacional y calidad de la docencia en la escuela de medicina. ARS Médica (Santiago) 2007; 15. URL: http://escuela.med.puc.cl/publ/arsmedica/arsmedica15/ ambiente.html.

4. Rothman AI, Ayoade F. The development of learning environment: a questionnaire for use in curriculum evaluation. J Med Educ 1970; 54: 754-9.

5. Marshall RE. Measuring the medical school learning environment. J Med Educ 1978; 53: 89-104.

6. Wakeford RE. Student's perception of the medical school learning environment: a pilot study into some differences and similarities between medical schools in the UK. Assessment and Evaluation in Higher Education 1981; 6: 206-17.

7. Roff S, McAleer S, Harden RM, Al-Qahtani M, Ahmed AU, Deza $\mathrm{H}$, et al. Development and validation of the Dundee Ready Education Environment Measure (DREEM). Med Teach 1997; 19: 295-9.

8. Roff S. The Dundee Ready Educational Environment Measure (DREEM) - a generic instrument for measuring students' perceptions of undergraduate health professions curricula. Med Teach 2005; 27: 322-5.

9. McAleer S, Roff S. Part 3: a practical guide to using the Dundee Ready Education Environment Measure (DREEM). In Genn JM, ed. Curriculum, environment, climate, quality and change in medical education: a unifying perspective. AMEE Education Guide n. ${ }^{\circ}$ 23. Dundee: Association of Medical Education in Europe; 2002.

10. Escanero JF, Mora S, Arce J, Bianchi R, Díaz-Véliz G, Gargiulo PA, et al. Estilos de aprendizaje y currículum: propuestas de mejora. Zaragoza: Prensas Universitarias de Zaragoza; 2009.

11. Pimparyon P, Roff S, McAleer S, Poonchai B, Pemba S. Educational environment, student approaches to learning and academic achievement in a Thai nursing school. Med Teach 2000; 22: 359-64.

12. Roff S, McAleer S, Ifere OS, Bhattacharya S. A global diagnostic tool for measuring educational environment: comparing Nigeria and Nepal. Med Teach 2001; 23: 378-82.
13. Bassaw B, Roff S, McAleer S, Roopnarinesingh S, De Lisle J, Teelucksingh S, et al. Students' perspectives on the educational environment, Faculty of Medical Sciences, Trinidad. Med Teach 2003; 25: 522-6.

14. Al-Ayed IH, Sheik SA. Assessment of the educational environment at the College of Medicine of King Saud University, Riyadh. East Mediterr Health J 2008; 14: 953-9.

15. Till H. Climate studies: can students' perceptions of the ideal educational environment be of use for institutional planning and resource utilization? Med Teach 2005; 27: 332-7.

16. Lempp $\mathrm{H}$, Seale $\mathrm{C}$. The hidden curriculum in undergraduate medical education: qualitative study of medical students' perceptions of teaching. BMJ 2004; 329: 770-3.

17. Irby DM. Clinical teaching and the clinical teacher. J Med Educ 1986; 35: 45.

18. Mangione CM. How medical school did not prepare me for graduate medical education. J Med Educ 1986; 61: 3-10.

19. Barrows HS. The scope of clinical education. J Med Educ 1986; 61: 23-33.

20. Miles S, Leinster SJ. Comparing staff and student perceptions of the student experience at a new medical school. Med Teach 2009; 31: 539-46.

21. Jiffry MTM, McAleer S, Fernando S, Maradinghe RB. Using the DREEM questionnaire to gather baseline information on an evolving medical school in Sri Lanka. Med Teach 2005; $27: 348-52$.

22. De Oliveira Filho GR, Vieira JE, Schonhorst L. Psychometric properties of the Dundee Ready Educational Environment Measure (DREEM) applied to medical residents. Med Teach 2005; 27: 343-7.

23. Al-Hazmi A, Al-Hyiani A, Roff S. Perceptions of the educational environment of the medical school in King Abdul Aziz University, Saudi Arabia. Med Teach 2004; 26: 570-3.

24. Till $\mathrm{H}$. Identifying the perceived weaknesses of a new curriculum by means of the Dundee Ready Education Environment Measure (DREEM) Inventory. Med Teach 2004; 26: 39-45. 